\title{
Gastroenteropancreatic Neuroendocrine Tumors (GEPNET) Registry 2009-2018: A Large Multi- country Registry with Over 1000 GEPNET Patients
}

\section{Suayib Yalcin ( $\nabla$ syalcin@hacettepe.edu.tr)}

Hacettepe University, Institute of Cancer

Hande Turna

Istanbul University Cerrahpaşa

llan Shimon

Rabin Medical Center, Beilinson and Sackler Faculty of Medicine, Tel-Aviv University

Yi-Ming Shyr

Taipei Veterans General Hospital

Yan-Shen Shan

National Cheng Kung University Hospital

Nuri Faruk Aykan

Istanbul University

\section{Sezer Saglam}

Demiroglu Bilim University, Group Florence Nightingale Hospital

Ahmet Sezer

Başkent University

Perran Fulden Yumuk

Marmara University

Jose Ramos

Donald Gordon Medical Centre

Kun-Huei Yeh

National Taiwan University Cancer Center and National Taiwan University Hospital

Colette Hanna

Saint Joseph Hospital

Hwee Yong Lim

National Cancer Centre Singapore

Vajarabhongsa Bhudhisawasdi

Khon Kaen University

Gokmen Aktas

Kahramanmaraş Sütçü İmam University

Li-Yuan Bai 
China Medical University Hospital

\section{Hussein Raef}

King Faisal Specialist Hospital \& Research Centre

\section{Tai-Jan Chiu}

Kaohsiung Chang Gung Memorial Hospital

\section{Mike Lau}

Novartis (Switzerland)

\section{Cassandra Slader}

Novartis (Switzerland)

\section{Tsann-Long Hwang}

Linkou Chang Gung Memorial Hospital

\section{Research Article}

Keywords: Gastroenteropancreatic neuroendocrine tumor, observational study, registry, diagnosis, treatment patterns

Posted Date: September 1st, 2021

DOI: https://doi.org/10.21203/rs.3.rs-827684/v1

License: (c) (1) This work is licensed under a Creative Commons Attribution 4.0 International License. Read Full License 


\section{Abstract}

Background: This 8-year observational, multicenter, registry study was undertaken to assess the incidence and regional trends in diagnosis, clinical management, and outcome assessments of patients with gastroenteropancreatic neuroendocrine tumors (GEP-NET) in the Asia-Pacific region, the Middle East, Turkey, and South Africa.

Materials and Methods: Patient data including demographics, primary tumor site, tumor differentiation, diagnosis, treatment modalities, outcomes were collected via a web-based data entry portal.

Results: Overall, 974 patients were enrolled, and 951 of which were evaluated (median age: 54 years). Pancreas was the most commonly reported primary tumor site. Non-functioning and functioning (most common: carcinoid) tumors were reported in $61.9 \%$ and $27.9 \%$ of patients, respectively. Abdominal pain was the most commonly reported symptom. Surgery was the most-commonly employed initial treatment, followed by treatment with somatostatin analogs; $>80 \%$ of patients had open surgery and R0 resection. Satisfactory response to the initial treatment modality was reported for $56.4 \%$ of patients. Overall survival rate was $96.3 \%$ at 6 months since diagnosis and $72.9 \%$ at 60 months. Median progression-free survival was 60.9 months since first diagnosis and 60.5 months after first treatment, with a 6-month progressionfree survival of $95.0 \%$.

Conclusion:The GEP-NET registry provides important information relating to the diagnosis and treatment of patients in the participating countries. This analysis highlighted the need for improvements in the clinical practice to ensure better evaluation and treatment management.

\section{Introduction}

Neuroendocrine tumors (NET) are rare, usually slow-growing heterogeneous malignancies originating from neuroendocrine cells found throughout the body [1, 2]. According to the United States (US) population-based Surveillance, Epidemiology, and End Results (SEER) registry, the incidence and prevalence of NET have risen steeply over the past 3 decades from 1.1 per 100000 in 1973 to 7.0 per 100 000 in 2012, and it continues to grow, regardless of tumor nature [3]. This increase in incidence of NET may be attributed to improved diagnostic methods and disease awareness over time [4, 5]. Unlike the increase in the incidence and prevalence observed in SEER, there is no statistically significant difference in overall survival (OS) duration among patients with local and regional NETs over time, although the OS rates have improved by $21.3 \%$ in patients diagnosed during 2009-2012 compared with 2000-2004 [3].

Among NET, gastroenteropancreatic (GEP) NET are the most prevalent $[1,6,7]$. According to the US SEER 18 grouping (2000-2012), the incidence of GEP-NET was 3.6/100 000 [3]. GEP NETs are noted for their lack of defining symptoms, which may lead to a delay in diagnosis $[2,8-10]$. However, upon metastasis, patients may develop debilitating symptoms associated with the release of highly bioactive substances. These symptoms of metastasis (referred to as carcinoid syndrome) are associated with significant morbidity, and are often vague that misdiagnoses in the form of more common disorders, such as 
irritable bowel syndrome, are common. The misdiagnosis puts carcinoid syndrome patients at a higher risk for carcinoid crises [2].

In the Asia-Pacific and Middle East regions as well as in Turkey and South Africa, there is a paucity of published information on the epidemiology and treatment of GEP-NETs [11-13]. In addition, there is a lack of consensus on the diagnosis and treatment of GEP-NETs in these areas which may contribute to the late diagnosis, inadequate treatment, underestimation of GEP-NET prevalence and inability to prioritize treatment from the available options. Hence, the current study examined the incidence and regional trends in diagnosis, clinical management, and outcome assessments of patients with GEP-NET in countries participating in the GEP-NET Registry from Asia-Pacific, Middle East, Turkey, and South Africa.

\section{Materials And Methods}

\section{Study Design}

The GEP-NET Registry was a longitudinal (8 years), observational, multicenter registry in participating countries. Patient data, including demographics, primary tumor site, tumor differentiation, diagnosis, treatment modalities, and outcomes, were collected via a web-based data entry portal retrospectively and prospectively. There were no mandated visits in this registry; however, it was recommended that relevant data regarding each patient be entered, as it emerged in order to optimize efficiency and accuracy. For each patient enrolled, there was a minimum requirement of 2 data collections per year, at 6-month intervals, starting from baseline visit of each patient for up to 5 years, to ensure an ongoing data stream. The initial enrollment period was anticipated to be about 36 months with a follow-up observational period of 5 years.

\section{Study Outcomes}

The primary objective of the study was to assess regional trends in the diagnosis, practical clinical management and outcome measures of patients with GEP-NET in participating countries.

Secondary objectives included the description of clinical basis of GEP-NET diagnosis, site of surgery, local ablative therapy, radiotherapy, nuclear medicine, somatostatin analogs, chemotherapy, and novel targeted therapy in practical clinical management of GEP-NET, parameters utilized to evaluate tumor response and disease biochemical response in clinical practice; assessment of the incidence of familial syndromes with GEP-NET, OS and progression-free survival (PFS) of patients with GEP-NET.

\section{Patient Population}

Patients with confirmed histopathological diagnosis of GEP-NET within 5 years prior to registry entry were included. Patients, who were unlikely to be able to provide long-term follow-up information for reasons of 
unavailability or severe concomitant illnesses, as per investigator's opinion, or simultaneous registration at another participating site of the GEP-NET registry, were excluded.

\section{Study Variables}

Baseline data collection included patient demographics, and historical data about the primary and eligible diagnosis and certain aspects of the patients' medical history. If applicable, it also included both the recordings of relevant historical management prior to registry entry, as well as the current disease status and management strategies. After baseline data collection, the follow-up data included ongoing patients', disease status, methods used to evaluate ongoing response, treatment changes and development of other cancers or familial GEP-NET. All assessments were based on the 2000 World Health Organization (WHO) classification as the study was initiated in 2009.

The date of first treatment was captured from the eCRF medication or surgery forms (whichever was earlier) triggered by the "Diagnosis of the first GEP-NET" form. Baseline was defined as the date when a patient provided the informed consent form. The last visit date was defined as the date of death, date of discontinuation from the registry, or the last date of data entry or data reported. The end of enrollment was defined as 36 months after the first patient was enrolled. If a patient had no valid first treatment date (the patients without surgery or medication information), the date of first treatment date was categorized as the first diagnosis date.

\section{Statistical Analysis}

Since there was no hypothesis, there was no formal sample size determination and the statistical analysis was largely descriptive. Descriptive analyses were performed using SAS $\circledast$ version 9.1.3 in a secure and validated environment. The data were summarized with respect to demographic and baseline characteristics, diagnostic and follow-up measures, disease stage, treatment patterns, dose optimization, and effectiveness. Dose/regimen changes, reasons for dose/regimen change, time from surgery, and treatment duration were summarized. Tumor response that was reported as either satisfactory or unsatisfactory for the measurement of treatment effectiveness was summarized. The incidence of recurrence, disease progression and death, and the aggregate variable of these incidences were calculated. The OS and PFS were analyzed using the Kaplan-Meier method, and the data censoring issues were incorporated for patients who withdrew from the registry. The missing data values were not imputed. The descriptive statistics were also provided for relevant characteristics of the eligible diagnosis and how it was managed, practices of primary and secondary genetic mutational analysis, and development of other cancers and familial GEP-NET. Each outcome was summarized for all patients combined and according to specific subgroups (eg, treatment modality, region, country, center, etc).

\section{Results}


From August 2009 to March 2018, 1028 patients were screened, and 974 patients (94.7\%) who met all the eligibility criteria were enrolled into the registry. Of those, 951 patients $(97.6 \%)$ were considered evaluable. Numbers of evaluable patients recruited per country are presented in Supplementary Table 1. Among enrolled patients, 638 (65.5\%) did not complete the planned observational period. Of those $306(31.4 \%)$ patients were lost to follow-up, $260(26.7 \%)$ died, 47 (4.8\%) discontinued for other reasons, 24 (2.5\%) withdrew consent, and $1(0.1 \%)$ enrolled in another clinical trial resulting in exclusion from this concurrent GEP-NET registry participation. Median duration of follow-up was 38.7 months (range: $0-88$ months). Patient demographics are shown in Table 1. Median age of evaluable patients at the time of obtaining informed consent was 55 years (range: 12-87 years), and median age at the time of first diagnosis of GEP-NET was 54 years (range: 12-87 years). There were similar numbers of males (471 patients [49.5\%]) and females (480 patients [50.5\%]). Majority were Caucasians (551 patients [57.9\%]), followed by Asians (366 patients [38.5\%]), Africans (21 patients [2.2\%]), and other races (13 patients [1.4\%]).

Table 1

Primary Tumor Site by WHO Classification

\begin{tabular}{|llllll|}
\hline Site & $\begin{array}{l}\text { Overall } \\
\mathbf{N}= \\
\mathbf{9 5 1}\end{array}$ & $\begin{array}{l}\text { Well- } \\
\text { differentiated } \\
\text { endocrine } \\
\text { tumor }\end{array}$ & $\begin{array}{l}\text { Well- } \\
\text { differentiated } \\
\text { endocrine } \\
\text { carcinoma }\end{array}$ & $\begin{array}{l}\text { Poorly } \\
\text { differentiated } \\
\text { endocrine } \\
\text { carcinoma }\end{array}$ & $\begin{array}{l}\text { Mixed exocrine- } \\
\text { endocrine } \\
\text { carcinoma }\end{array}$ \\
\hline $\begin{array}{l}\mathrm{n}=\mathbf{3 2 8} \\
\mathrm{Pancreas,} \\
\mathrm{n}(\%)\end{array}$ & $\begin{array}{l}408 \\
(42.9)\end{array}$ & $149(45.4)$ & $162(44.1)$ & $41(33.6)$ & $2(22.2)$ \\
\hline $\begin{array}{l}\text { Stomach, } \mathrm{n} \\
(\%)\end{array}$ & $\begin{array}{l}163 \\
(17.1)\end{array}$ & $71(21.6)$ & $36(9.8)$ & $31(25.4)$ & $3(33.3)$ \\
\hline $\begin{array}{l}\text { Colon- } \\
\text { rectum, } \mathrm{n} \\
(\%)\end{array}$ & $\begin{array}{l}106 \\
(11.1)\end{array}$ & $31(9.5)$ & $45(12.3)$ & $16(13.1)$ & $1(11.1)$ \\
\hline $\begin{array}{l}\text { Jejuno- } \\
\text { ileum, } \mathrm{n} \\
(\%)\end{array}$ & $\begin{array}{l}65 \\
(6.8)\end{array}$ & $11(3.4)$ & $45(12.3)$ & $4(3.3)$ & 0 \\
\hline $\begin{array}{l}\text { Duodenum, } \\
\mathrm{n}(\%)\end{array}$ & $\begin{array}{l}50 \\
(5.3)\end{array}$ & $19(5.8)$ & $19(5.2)$ & $7(5.7)$ & $1(11.1)$ \\
\hline $\begin{array}{l}\text { Appendix, } \\
\mathrm{n}(\%)\end{array}$ & $\begin{array}{l}39 \\
(4.1)\end{array}$ & $16(4.9)$ & $16(4.4)$ & $1(0.8)$ & $2(22.2)$ \\
\hline $\begin{array}{l}\text { Others, } \mathrm{n} \\
(\%)\end{array}$ & $\begin{array}{l}120 \\
(12.6)\end{array}$ & $31(9.5)$ & $44(12.0)$ & $22(18.0)$ & 0 \\
\hline WHO, World Health Organization. & & & 0 \\
\hline
\end{tabular}

Among those who were evaluable, 100 patients (10.5\%) had a history of other non-GEP-NET malignancies, and most common types of non-GEP-NET malignancies in these patients were thyroid (14 patients [14\%]), lower gastrointestinal region (13 patients [13\%]), and breast carcinomas (10 patients 
[10\%]). Only 18 patients (1.9\%) had a family history (parents or siblings) of GEP-NET, 774 patients (81.4\%) had no family history, and it was unknown in 159 patients (16.7\%).

\section{Diagnosis and Symptoms of the First GEP-NET}

Tumor tissue pathology was the most common method (99.6\%) used to diagnose GEP-NET, followed by computerized tomography (CT) scans (52.4\%) (Fig. 1). Median number of measurement methods used for diagnosing GEP-NET in a single patient was 2.0 (range, 1-7). Most common primary site of tumor was pancreas $(n=408 ; 43 \%)$, followed by stomach $(n=163 ; 17 \%)$ (Table 1$)$. By pathological confirmation of the primary diagnosis, 265 patients $(27.9 \%)$ had a functioning GEP-NET, including carcinoid $(63.8 \%)$, insulinomas (17.4\%), gastrinomas (11.7\%), VIPomas (0.8\%), glucagonomas $(1.5 \%)$, somatostatinomas (1.9\%), and others (4.9\%); 589 patients (61.9\%) presented with nonfunctioning GEP-NET, and 25 patients $(2.6 \%)$ had unknown primary. Pathologic confirmation was unknown/not applicable in 72 patients (7.6\%) (Fig. 2A).

At diagnosis, 740 patients (77.8\%) had at least 1 symptom for the first GEP-NET. Most commonly reported symptoms were abdominal pain, weight loss, and diarrhea (Fig. 2B). Among patients with functioning and nonfunctioning GEP-NET the percentage of symptom burden is shown in Table 2.

Table 2

Symptom Burden across GEP-NET Patients

\begin{tabular}{|lll|}
\hline Number of Symptoms & Functioning GEP-NET (\%) & Non-functioning GEP-NET(\%) \\
\hline No symptoms & 15.1 & 23.3 \\
\hline At least 1 symptom & 38.1 & 41.4 \\
\hline 2 symptoms & 23.4 & 21.2 \\
\hline 3 symptoms & 14.0 & 8.8 \\
\hline 4 symptoms & 5.7 & 2.7 \\
\hline$\geq 5$ symptoms & 3.8 & 2.5 \\
\hline GEP-NET, gastroenteropancreatic neuroendocrine tumor.
\end{tabular}

\section{Initial GEP-NET Treatment}

Different modalities employed for the initial treatment of patients with GEP-NET were surgery $(n=573$; $60.3 \%)$, somatostatin analogs $(n=161 ; 16.9 \%)$, chemotherapy $(n=143 ; 15.0 \%)$, radiotherapy $(n=33$; $3.5 \%)$, embolization $(n=31 ; 3.3 \%)$, other medication $(n=13 ; 1.4 \%)$, alpha interferon therapy $(n=3 ; 0.3 \%)$, and other treatment modalities $(n=107 ; 11.3 \%)$ (Fig. 3A). Among the patients who had undergone at least one surgery at baseline for the purpose of initial diagnosis $(59.8 \%)$, the goal of surgery was achieved in $86.3 \%$ (Fig. 3B). After the initial treatment, 536 patients $(56.4 \%)$ had a satisfactory tumor 
response or stable disease, while 84 patients $(8.8 \%)$ had no or lack of tumor response. Various tests were used in 667 (70.1\%) patients for assessing tumor response to initial treatment of GEP-NET. Most commonly used assessment method was CT scan, which was performed in 509 patients (76.3\%) (Fig. 3C).

\section{Treatment and Clinical Status of GEP-NET at Baseline}

A total of 569 patients (59.8\%) had surgery at least once at baseline in order to diagnose initially; 399 (70.1\%), 66 (11.6\%), and 58 patients (10.2\%) underwent R0, R1, and R2 resections, respectively. Proportion of patients who had a tumor tissue pathology test at baseline was 946 (99.5\%). Most common tumor tissue pathology reported was well-differentiated endocrine carcinomas in 367 patients (38.8\%), followed by well-differentiated endocrine tumor in 328 patients (34.7\%). Poorly differentiated and mixed exocrine-endocrine carcinomas were observed in 122 (12.9\%) and 9 patients (1.0\%), respectively. Highly prevalent types among functional GEP-NET through tumor tissue pathology at baseline were gastrinomas (7.6\%) and insulinomas (5.3\%), while $81.8 \%$ of patients had nonfunctional GEP-NET. Chromogranin A ( $\mathrm{CgA})$ and synaptophysin were the best-tested tumor markers with $\mathrm{CgA}$ positive in 677 patients (71.6\%) and synaptophysin positive in 692 patients (73.2\%) (Table 3).

Table 3

Tumor Tissue Pathology at Baseline

\begin{tabular}{|c|c|c|c|}
\hline & \multicolumn{3}{|l|}{$N=951$} \\
\hline $\begin{array}{l}\text { Number of patients who had tumor tissue pathology } \\
\text { test, } \mathrm{n}(\%)\end{array}$ & \multicolumn{3}{|c|}{$946(99.5)$} \\
\hline \multicolumn{4}{|l|}{ Tumor markers, n (\%) } \\
\hline & $\mathrm{CgA}$ & Synaptophysin & NSE \\
\hline Positive & $\begin{array}{l}677 \\
(71.6)\end{array}$ & $692(73.2)$ & $112(11.8)$ \\
\hline Negative & $89(9.4)$ & $26(2.7)$ & $17(1.8)$ \\
\hline Not done & $\begin{array}{l}179 \\
(18.9)\end{array}$ & $227(24.0)$ & $816(86.3)$ \\
\hline Missing & $1(0.1)$ & $1(0.1)$ & $1(0.1)$ \\
\hline \multicolumn{4}{|l|}{ Proliferative markers, n (\%) } \\
\hline & \multicolumn{2}{|c|}{ Ki-67, n (\%) } & $\begin{array}{l}\text { Mitotic index } \\
\mathrm{n}(\%)\end{array}$ \\
\hline Done & \multicolumn{2}{|c|}{$476(50.3)$} & $193(20.4)$ \\
\hline Not done & \multicolumn{2}{|c|}{$464(49.0)$} & $367(38.8)$ \\
\hline
\end{tabular}




\section{Overall Survival and Progression-Free Survival}

Estimated median OS was not reached. Rate of OS at 6 months was $96.3 \%$, and $72.9 \%$ in 60 months Patients with poorly differentiated tumors showed worse survival with OS rates of: $90.2 \%$ in 6 months and $46.4 \%$ in 60 months; PFS rates of: $90.2 \%$ in 6 months and $31 \%$ in 60 months. While, welldifferentiated tumors showed better survival (with 6 months OS rate of $98.8 \%$, and 60 months of $82.3 \%$; and 6 months PFS rate of $93.3 \%$, and 60 months of $41.2 \%$ ). The overall survival by primary tumor site and WHO classification are presented in Figs. 4A and 4B. Median time for the PFS was 60.9 months from the first diagnosis for the whole study population. Six months PFS rate was $95.0 \%$, and at 60 months PFS rate was $50.4 \%$.

\section{Safety}

Number of patients with at least 1 adverse event was 56 (5.9\%), and 21 patients (2.2\%) had at least 1 serious adverse event. During the course of the study, 270 deaths $(28.4 \%)$ were reported, with 209 (77.4\%) of these were related to GEP-NET.

\section{Discussion}

This GEP-NET registry study aimed to assess the incidence, and regional trends in diagnosis, practical clinical management, and outcome measures of patients with GEP-NET in the Asia-Pacific region, South Africa, Turkey and the Middle East. Clinical basis of GEP-NET diagnosis, different therapies in practical clinical management of GEP-NET, parameters utilized to evaluate the tumor response and disease biochemical response in the clinical practice, OS, and PFS were also evaluated, similar to that of the published literature. In this GEP-NET registry, the most common primary tumor site was the pancreas; nonfunctioning, well-differentiated endocrine carcinoma was the most common type of tumor tissue pathology. Based on the SEER database analysis of patients with neuroendocrine tumors, the lung/bronchus was the most common site of NETs with $30.6 \%$, while pancreas accounted for $10.8 \%$ of NETs [14]. It was earlier reported that the patients with NETs in the pancreas had the highest risk of mortality [14], and in the current study patients with GEP-NET with pancreas as the primary tumor site showed median PFS of 74.6 months. Consistent with the earlier published literature, patients with poorly differentiated endocrine carcinomas had poor survival outcomes in the present registry [15].

In this registry, surgery was the most commonly employed initial treatment for GEP-NET followed by systemic therapy with somatostatin analogs and more than $80 \%$ of patients had R0 resection and open surgeries preferred over laparoscopic surgeries. Best chance for cure, symptomatic relief, and/or longterm survival is the success of the surgical resection of the primary tumor, regional nodal disease and distant metastases $[16,17]$. Surgery is recommended treatment in patients with GEP-NETs of any stage, especially if primary tumor can be removed and $70-90 \%$ of its metastasis can be debulked [17-19].

It was previously reported that CT scan was the first assessment of suspected GEP-NET [20]. In this registry, most commonly used method for the initial diagnosis of GEP-NET and for the assessment of 
tumor response post the initial treatment was also CT scan. Current study also revealed poor availability of specific diagnostic methods, such as CgA testing, positron emission tomography (PET) or octreotide scans, at different centers, which is likely to represent the difference in the accessibility of these tests in different locations. Eventual decline in the rate of OS and PFS over 60 months in the current registry further demonstrated that despite overall favorable prognosis, tumors continue to progress and eventually lead to death in large number of patients, especially in patients with poorly differentiated histology. Baseline TNM staging information was not available for all patients; hence, current study could not delineate treatment and outcomes by stage of the disease. Furthermore, survival outcomes were not evaluated based on surgery outcome status at the start of the study.

The strength of this study is the large patient population of 951 patients from over 70 centers in 15 countries across various geographical regions (Africa, Middle East, and Asia), which reinforces the external validity/generalizability of the results in the emerging countries.

Observational studies in any therapeutic area are subject to inherent methodological and operational limitations. For example, the data availability, and hence, completeness is highly variable as usual medical care can differ among physicians, sites, and countries. In this non-interventional study, to minimize the overall bias, the study involved many centers across many countries. Since this study was designed in 2009, the comparison of these results to current data based on the updated "WHO classification criteria" is not appropriate. Besides, unavailability of WHO classified data is an added limitation to this study

Since WHO guidelines have been updated in 2010 and 2017, use of 2000 WHO guidelines is a limitation of the study. Hence, comparison of the results from this study with current data should be done cautiously.

\section{Conclusion}

To summarize, pancreas was the most common primary site; nonfunctioning, well-differentiated endocrine carcinoma was the most common type of tumor grade; and patients with poorly differentiated neuroendocrine carcinoma had poor survival outcomes. This study shows the lack of availability of specific diagnostics methods such as CgA testing, PET or octreotide scans at different centers, which is likely to represent the difference in accessibility of these tests in different locations. It also demonstrates that in spite of overall favorable prognosis, tumors continue to progress and eventually lead to death in large number of patients, especially in patients with poorly differentiated histology. This analysis has highlighted the need for clinical practice improvement and outcome assessments to ensure better evaluation and management of patients with GEPNET in participating countries from Asia-Pacific, Middle East, Turkey, and South Africa.

\section{Abbreviations}


$\mathrm{CgA}$, chromogranin A; CT, computed tomography; GEP, gastroenteropancreatic; GEP-NET, gastroenteropancreatic neuroendocrine tumor; NET, neuroendocrine tumor; OS, overall survival; PFS, progression-free survival; PET, positron emission tomography; SEER, Surveillance, Epidemiology, and End Results; TNM, Tumor nodes and metastases; US, United States of America; WHO, World Health Organization.

\section{Declarations}

\section{Ethics approval and consent to participate}

The research was conducted ethically in accordance with the World Medical Association Declaration of Helsinki International Conference on Harmonisation Good Clinical Practice, patient privacy requirements, and ethical principles outlined in the Declaration of Helsinki 2018, and was first approved by IRB (reference number: UW 09-254) on the protocol v1 dated 15 June 2009. Health authority approval was obtained per local regulations. Patients provided informed consent according to local requirements.

\section{Consent for publication:}

Not applicable

\section{Availability of data and materials}

"Novartis is committed to sharing with qualified external researchers, access to patient-level data and supporting clinical documents from eligible studies. These requests are reviewed and approved by an independent review panel on the basis of scientific merit. All data provided is anonymised to respect the privacy of patients who have participated in the trial in line with applicable laws and regulations.

This trial data availability is according to the criteria and process described on www.clinicalstudydatarequest.com."

\section{Competing interests}

KHY reports personal fees from Ono Pharmaceutical Co. Ltd., Boehringer Ingelheim, Takeda, BMS, MSD, Eli Lilly, and Amgen, during the conduct of the study; HR reports grants from Novartis, during the conduct of the study; ML and CS are Novartis employees; SY, HD, IS, YMS, YSS, NFA, SS, AS, PFY, CH, HYL, VB, GA, LYB, TJC, TLH, declare that they have no competing interests.

\section{Funding}




\section{Author Contributions}

SY: Literature search, figures, study design, data collection, data analysis, data interpretation and writing/reviewing the manuscript; HD: Data collection, data interpretation and writing/reviewing the manuscript; IS: Data collection, data interpretation and writing/reviewing the manuscript; YMS: Literature search, data collection, data interpretation and writing/reviewing the manuscript; YSS: Literature search, figures, study design, data collection, data analysis, data interpretation and writing/reviewing the manuscript; NFA: Literature search, data interpretation and writing/reviewing the manuscript; SS: Data interpretation and writing/reviewing the manuscript; AS: Data collection, data interpretation, and writing/reviewing manuscript; PFY: Data collection, data interpretation, and writing/reviewing manuscript; JR: Data collection, data interpretation, and writing/reviewing manuscript; KHY: Data interpretation, and writing/reviewing manuscript; $\mathrm{CH}$ : Data interpretation, and writing/reviewing manuscript; $\mathrm{HYL}$ : Data interpretation, and writing/reviewing manuscript; VB: Data collection, data interpretation, and writing/reviewing manuscript; GA: Data collection, data interpretation, and writing/reviewing manuscript; LYB: Data collection, data interpretation, and writing/reviewing manuscript; HR: Data collection, data interpretation, and writing/reviewing manuscript; TJC: Data interpretation, and writing/reviewing manuscript; ML: Literature search, figures, data analysis, data interpretation, and writing/reviewing manuscript; CS: Study design, data collection, data analysis, data interpretation, and writing/reviewing manuscript; TLH: Literature search, figures, study design, data collection, data analysis, data interpretation, and writing/reviewing manuscript.

\section{Acknowledgement}

We thank the patients, caregivers, and investigators for their participation in the study. We also thank the worldwide network of research nurses, trial coordinators, and operations staff for their contributions. In addition, we would like to thank Ashwini Maratha, PhD, Anuradha Bandaru, PhD, of Novartis Healthcare Pvt. Ltd. for providing medical and editorial assistance with this manuscript.

\section{References}

1. Cives M, Strosberg JR. Gastroenteropancreatic Neuroendocrine Tumors. CA Cancer J Clin. 2018 Nov; 68(6): 471-487.

2. Modlin IM, Oberg K, Chung DC, Jensen RT, de Herder WW, Thakker RV, et al. Gastroenteropancreatic neuroendocrine tumours. Lancet Oncol. 2008 Jan; 9(1): 61-72.

3. Dasari A, Shen C, Halperin D, Zhao B, Zhou S, Xu Y, et al. Trends in the Incidence, Prevalence, and Survival Outcomes in Patients With Neuroendocrine Tumors in the United States. JAMA Oncol. 2017 Oct; 3(10): 1335-1342. 
4. Hendifar AE, Marchevsky AM, Tuli R. Neuroendocrine Tumors of the Lung: Current Challenges and Advances in the Diagnosis and Management of Well-Differentiated Disease. J Thorac Oncol. 2017 Mar; 12(3): 425-436.

5. Halperin DM, Shen C, Dasari A, Xu Y, Chu Y, Zhou S, et al. Frequency of carcinoid syndrome at neuroendocrine tumour diagnosis: a population-based study. Lancet Oncol. 2017 Apr; 18(4): 525534.

6. Yao JC, Hassan M, Phan A, Dagohoy C, Leary C, Mares JE, et al. One hundred years after "carcinoid": epidemiology of and prognostic factors for neuroendocrine tumors in 35,825 cases in the United States. J Clin Oncol. 2008 Jun; 26(18): 3063-3072.

7. Grozinsky-Glasberg S, Grossman AB, Korbonits $M$. The role of somatostatin analogues in the treatment of neuroendocrine tumours. Mol Cell Endocrinol. 2008 May; 286(1-2): 238-250.

8. O'Connor JM, Marmissolle F, Bestani C, Pesce V, Belli S, Dominichini E, et al. Observational study of patients with gastroenteropancreatic and bronchial neuroendocrine tumors in Argentina: Results from the large database of a multidisciplinary group clinical multicenter study. Mol Clin Oncol. 2014 Sep; 2(5): 673-684.

9. Díez M, Teulé A, Salazar R. Gastroenteropancreatic neuroendocrine tumors: diagnosis and treatment. Ann Gastroenterol. 2013; 26(1): 29-36.

10. Karpathakis A, Caplin M, Thirlwell C. Hitting the target: where do molecularly targeted therapies fit in the treatment scheduling of neuroendocrine tumours? Endocr Relat Cancer. 2012 May; 19(3): R73-92.

11. Pavel M, Öberg K, Falconi M, Krenning E, Sundin A, Perren A, et al. Gastroenteropancreatic Neuroendocrine Neoplasms: ESMO Clinical Practice Guidelines for Diagnosis, Treatment and Followup. Ann Oncol. 2020; 31(5): 0-0 Article in press.

12. Santos AP, Vinagre J, Soares P, Claro I, Sanches AC, Gomes L, et al. Gastroenteropancreatic Neuroendocrine Neoplasia Characterization in Portugal: Results from the NETs Study Group of the Portuguese Society of Endocrinology, Diabetes and Metabolism. Int J Endocrinol. 2019 Aug.

13. Iyer, R. and H. Hatoum. Highlights in GEP-NETs From the 13th Annual ENETS Conference for the Diagnosis and Treatment of Neuroendocrine Tumor Disease: Commentary. Clinical advances in hematology \& oncology: H\&O. 2016 Mar; 14(5 Suppl 7): 17.

14. Man D, Wu J, Shen Z, Zhu X. Prognosis of patients with neuroendocrine tumor: a SEER database analysis. Cancer Manag Res. 2018 Nov; 10: 5629-5638.

15. Sorbye H, Strosberg J, Baudin E, Klimstra DS, Yao JC. Gastroenteropancreatic high-grade neuroendocrine carcinoma. Cancer. 2014 Sep; 120(18): 2814-2823.

16. Givi B, Pommier SJ, Thompson AK, Diggs BS, Pommier RF. Operative resection of primary carcinoid neoplasms in patients with liver metastases yields significantly better survival. Surgery. 2006 Dec; 140(6): 891-7; discussion 897-898.

17. Hill JS, McPhee JT, McDade TP, Zhou Z, Sullivan ME, Whalen GF, et al. Pancreatic neuroendocrine tumors: the impact of surgical resection on survival. Cancer. 2009 Feb; 115(4): 741-751. 
18. Mayo SC, de Jong MC, Pulitano C, Clary BM, Reddy SK, Gamblin TC, et al. Surgical management of hepatic neuroendocrine tumor metastasis: results from an international multi-institutional analysis. Ann Surg Oncol. 2010 Dec; 17(12): 3129-3136.

19. Graff-Baker AN, Sauer DA, Pommier SJ, Pommier RF. Expanded criteria for carcinoid liver debulking: Maintaining survival and increasing the number of eligible patients. Surgery. 2014 Dec; 156(6): 136976; discussion 1376-1377.

20. Maxwell JE, O'Dorisio TM, Howe JR. Biochemical Diagnosis and Preoperative Imaging of Gastroenteropancreatic Neuroendocrine Tumors. Surg Oncol Clin N Am. 2016 Jan; 25(1): 171-194.

\section{Figures}

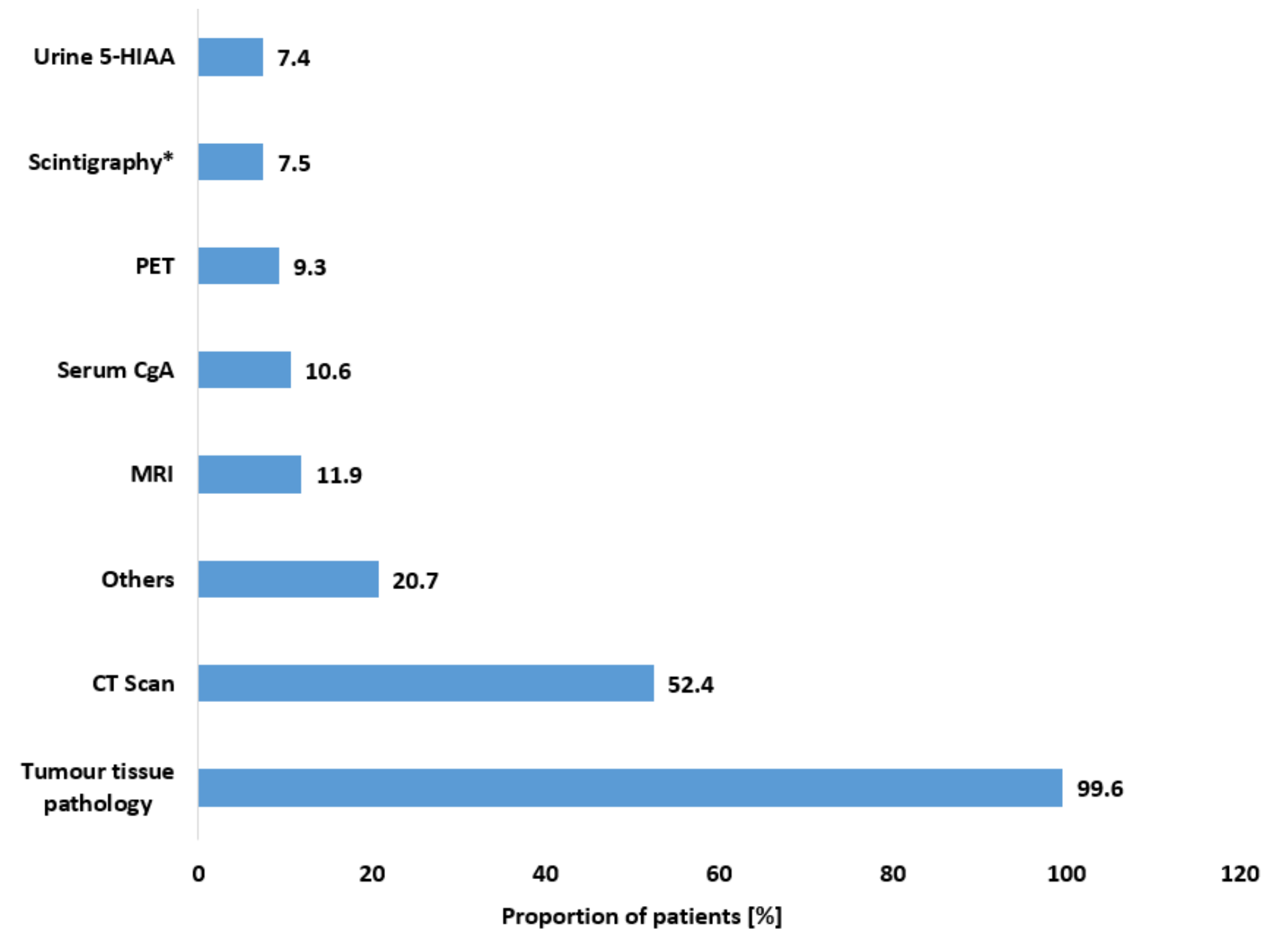

\section{Figure 1}

Test used for the first diagnosis of GEP-NET. *Includes octreotide scintigraphy performed in $5.6 \%$ patients. CgA, chromagranin A; CT, computerized tomography; HIAA, hydroxyindoleacetic acid; GEP-NET, 
gastroenteropancreatic neuroendocrine tumor; $\mathrm{MRI}$, magnetic resonance imaging; $\mathrm{PET}$, positron emission tomography; Scintigraphy, Somatostatin Receptor Scintigraphy and Octreotide Scintigraphy
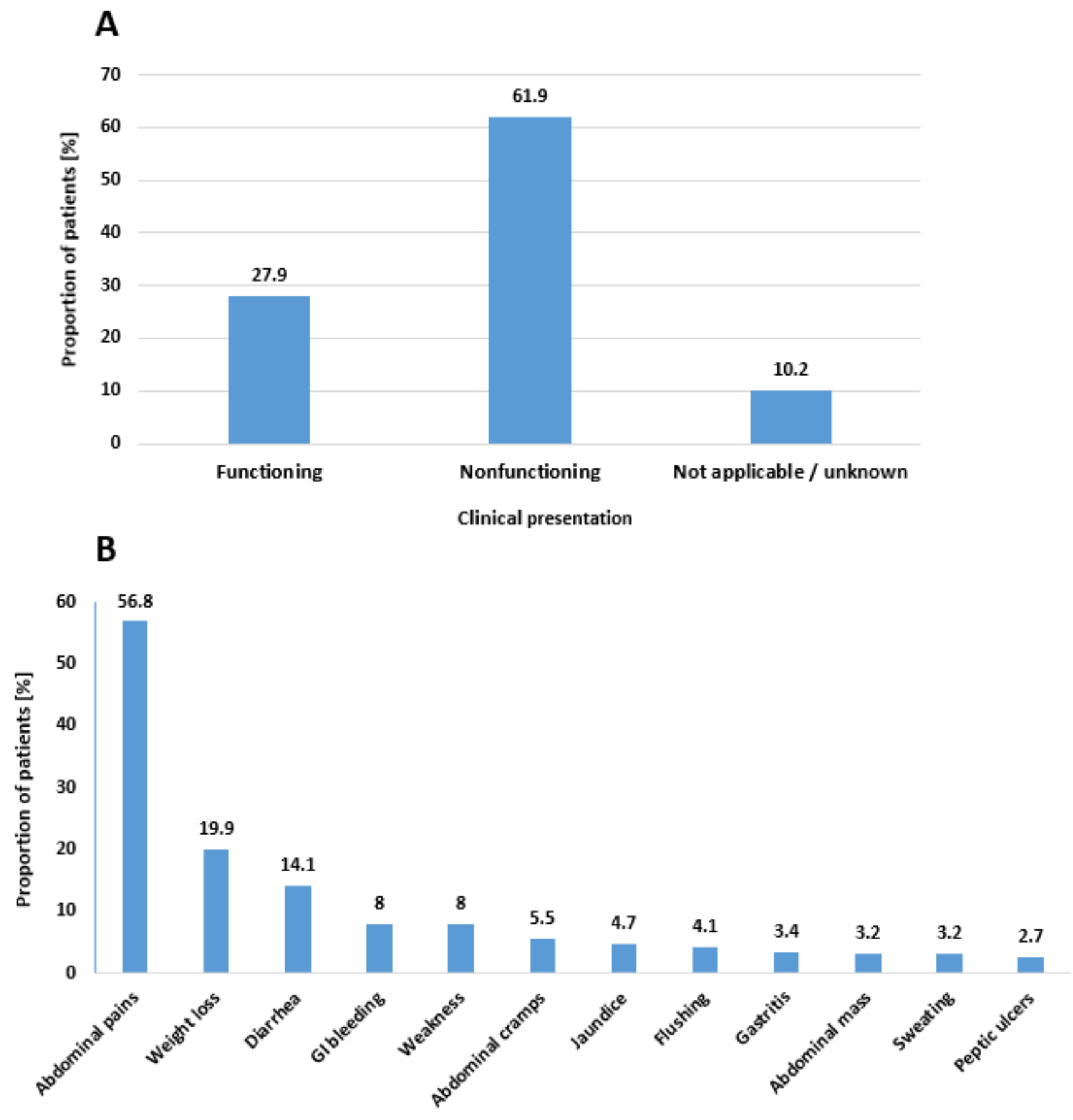

Type of symptoms

\section{Figure 2}

(A) Clinical presentation (B) Most common (> 2\%) symptoms reported in patients with first GEP-NET. GEPNET, gastroenteropancreatic neuroendocrine tumor; $\mathrm{Gl}$, gastrointestinal 
A

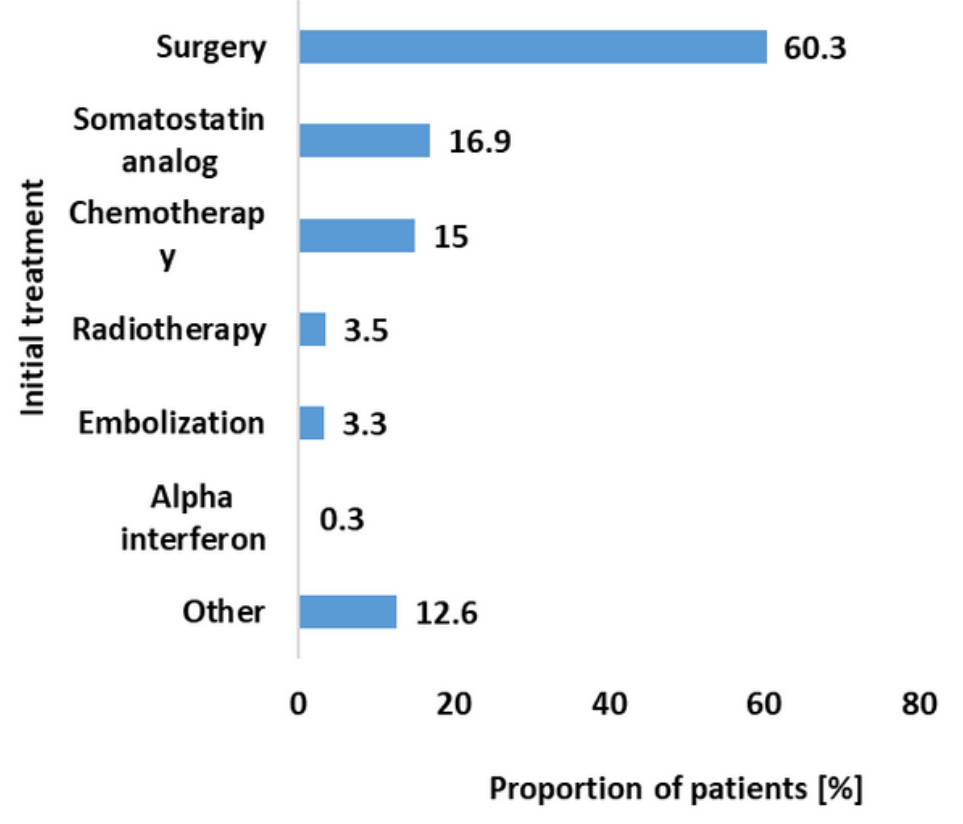

C

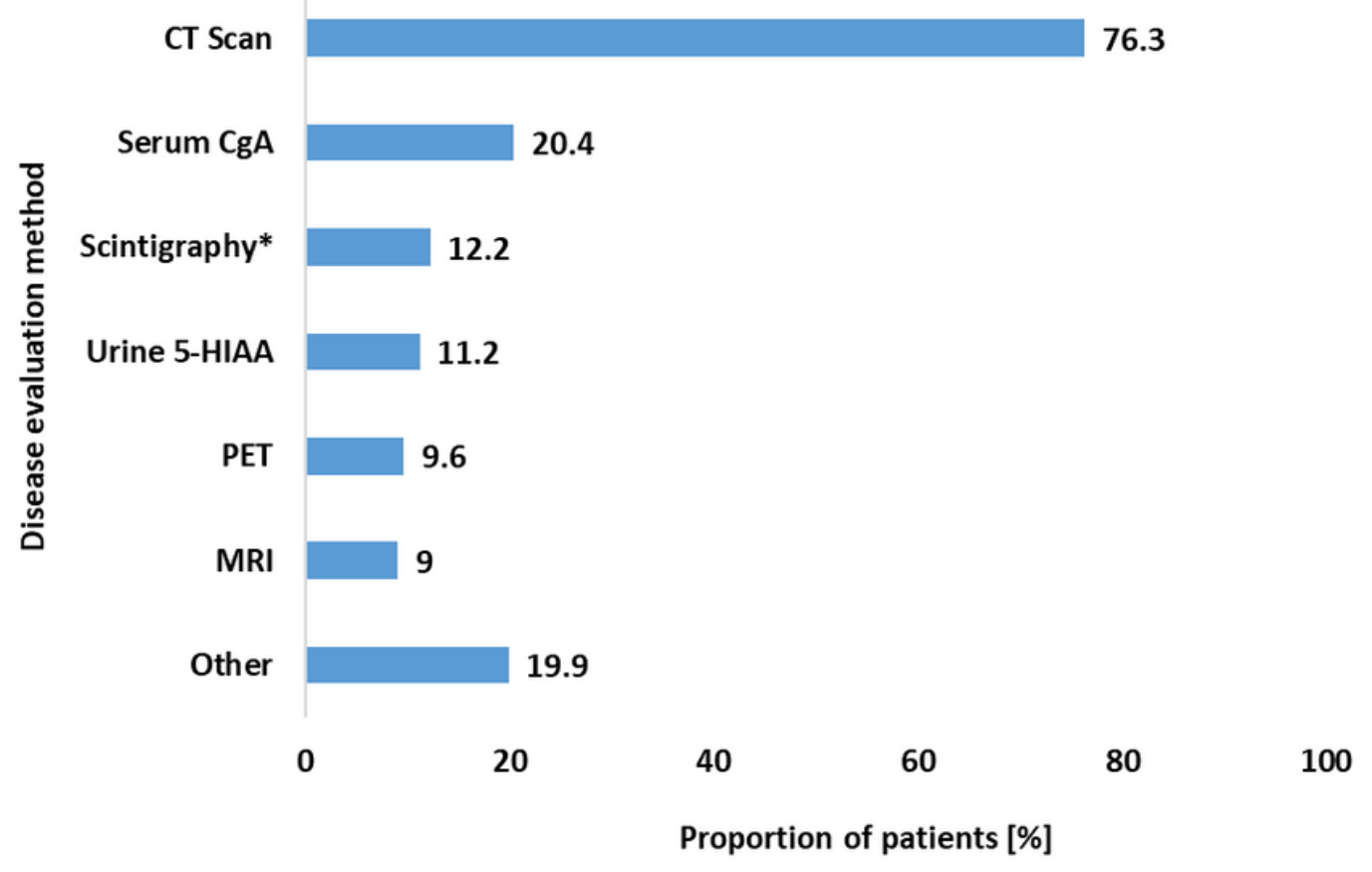

\section{Figure 3}

(A) Initial GEP-NET treatment (B) Surgery received for initial treatment (C) Disease evaluation methods employed. *Includes octreotide scintigraphy performed in $9.4 \%$ patients. $\mathrm{CgA}$, chromagranin A; CT, computerized tomography; HIAA; hydroxyindoleacetic acid; GEPNET, gastroenteropancreatic neuroendocrine tumor; MRI, magnetic resonance imaging; PET, positron emission tomography; Scintigraphy*, Somatostatin Receptor Scintigraphy and Octreotide Scintigraphy 
A

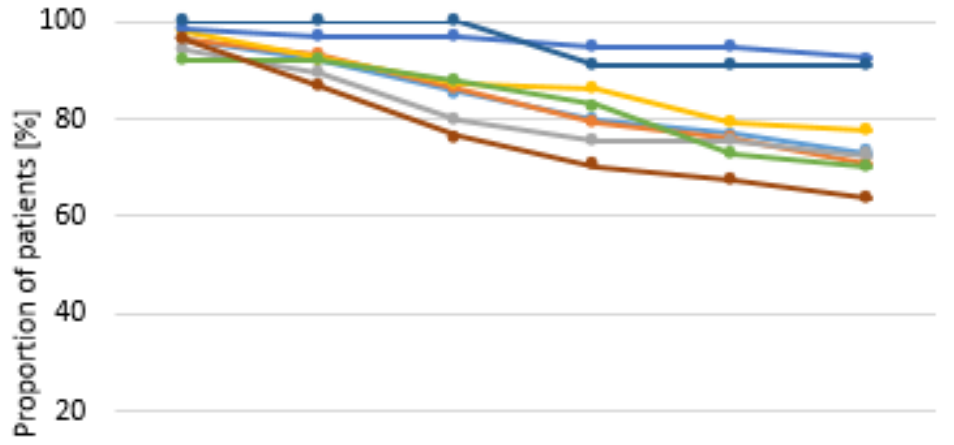

0

$\begin{array}{llllll}6 & 12 & 24 & 36 & 48 & 60\end{array}$

Time from first diagnosis [months]

-Total ( $n=951) \quad \longrightarrow$ Pancreas $(n=408)$

-Stomach ( $n=163) \quad$ Colon-rectum ( $n=106)$

- Jejunoileum $(n=65) \quad$ Duodenum $(n=50)$

*-Appendix $(n=39) \quad$ - Others $(n=119)$

B

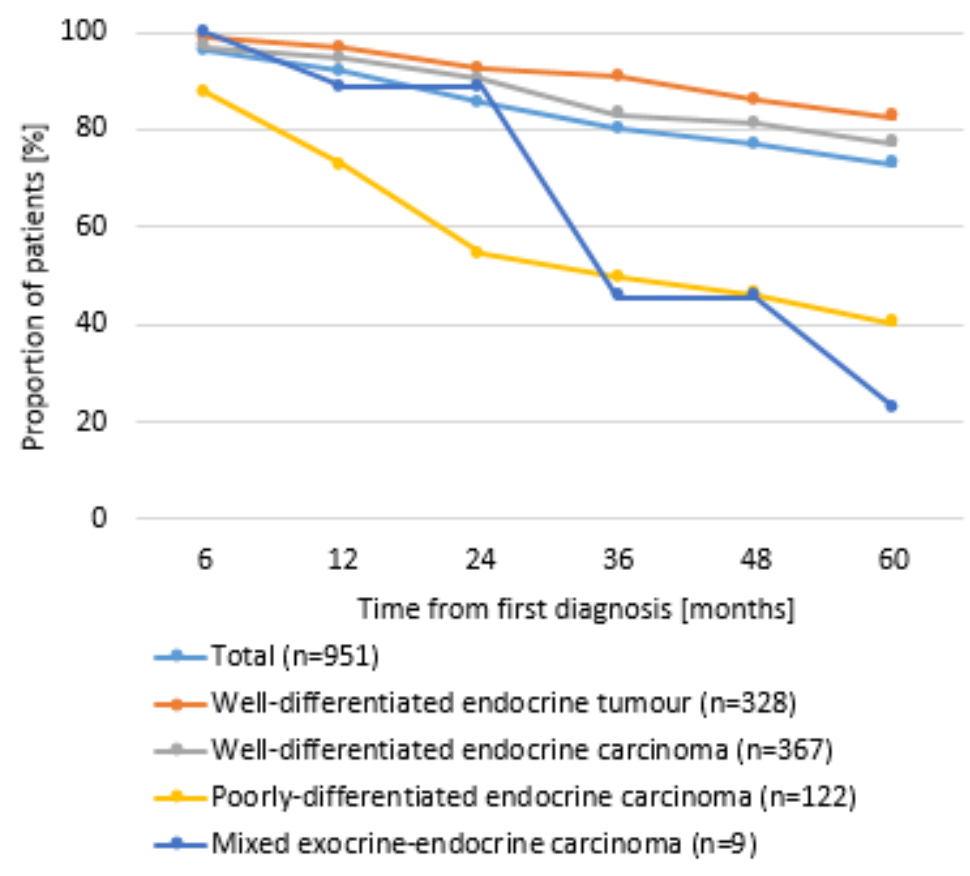

Figure 4

(A) Overall survival by primary tumor site (B) Overall survival by WHO classification. OS, overall survival; WHO, World Health Organization

\section{Supplementary Files}

This is a list of supplementary files associated with this preprint. Click to download.

- SupplementaryTable1.docx 